\author{
T.A. Grechukha1, M.G. Galitskaya ${ }^{1}$, A.G. Gayvoronskaya ${ }^{1}$, L.S. Namazova-Baranova ${ }^{1,2,3}$ \\ ${ }^{1}$ Scientific Center of Children's Health, Moscow, Russian Federation \\ ${ }^{2}$ First Sechenov Moscow State Medical University, Russian Federation \\ ${ }^{3}$ Pirogov Russian National Medical Research University, Moscow, Russian Federation
}

\title{
Rotavirus infection. How to really protect children from severe gastroenterites?
}

\section{Author affiliation:}

Grechukha Tat'yana Anatol'evna, MD, senior research scientist, immunologist of the vaccinal prevention department for children with health deviations of the $\mathrm{SCCH}$

Address: 2, Lomonosovskiy Av., Moscow, 119991, tel.: +7 (495) 967-14-20

Article received: 26.03.2013, accepted for publication:

According to the statistics of the recent 5 years, the share of rotavirus gastroenterites is $44-47 \%$ of all acute intestinal infections in children under 5 years of age in the Russian Federation. Up to $5 \%$ of mortality rate in children under 5 years of age is connected with rotavirus gastroenteritis. Rotavirus gastroenteritis takes an especially severe course in children of 6-24 months of age. The only reliable method of preventing this infection is vaccination. The authors present information on the rotavirus strains dominant in Russia and abroad, efficacy and safety of immunization with a pentavalent vaccine and the recommended schemes of its administration. This vaccine is registered in the Russian Federation; it is to be first used in the nearest future.

Keywords: rotavirus, strains, infants, vaccinal prevention, efficacy.

\section{Description of the causative agent}

Rotavirus belongs to family Reoviridae - non-enveloped icosahedral viruses. The virus takes its name after the Latin rota - wheel - due to its distinctive shape [1].

Rotavirus has a 3-layered structure: external capsid, internal capsid and nucleus. These layers envelop a double-stranded RNA consisting of 11 segments. Each segments of the genome encodes 1 viral protein.

Rotavirus consists of 6 structural (VP1-VP4, VP6, VP7) and 6 non-structural proteins (NSP1NSP6). 2 viral proteins - VP4 and VP7 - form the external envelope. Serotype-specific neutralizing antibodies form to each of the viral proteins. VP6 located in the internal capsid and VP2 located in the nucleus are antigenic determinants for rotavirus serogroups. VP4 is the main proteins, which ensures attachment of the virus to the host cell. Protein NPS4 has enterotoxin activity $[1,2]$.

Rotaviruses (RV) are divided into serogroups, subgroups, serotypes and genotypes. There are 6 serogroups, which definitely cause infections in mammals and birds. These serogroups differ in terms of antigens and are distinguished on the basis of specific determinants of structural proteins VP2 and VP6. Serogroup A has the highest clinical value. Rotaviruses of this serogroup are pathogenic to humans and animals and may cause gastroenteritis eruptions in all age groups.

Different serotypes of the virus are distinguished in serogroup A on the basis of neutralizing antibodies to superficial proteins VP4 and VP7. Division into serotypes $\mathrm{P}$ is based on the reaction with neutralizing antibodies to VP4 (a protease-split viral protein), division into serotypes $\mathrm{G}$ - on the reaction with neutralizing antibodies to VP7 (a glycoprotein antigen). Proteins VP4 and VP4 contain epitopes providing formation of serotype-specific neutralizing antibodies. As the genome's segment encoding VP7 characterizes G-serotype precisely, Gserotypes correspond to G-genotypes, while several P-genotypes may correspond to a P-serotype $[1,2]$.

At present, we know about 12 P-types and 11 G-serotypes, which may form 132 P-Gcombinations in humans. Strain P[8]G1 is the most widespread in the world. Ca. $50 \%$ of rotavirus gastroenterites (RVGE) in North America, South America and Europe are associated 
with strain $\mathrm{P}[8] \mathrm{G} 1$ (pic. 1); more than $30 \%$ of rotavirus infection cases in Africa and slightly less than $25 \%$ of cases in South-East Asia are associated with this strain [3].

The dominant rotavirus serotype changes from season to season in each country and may even be different in different regions of a country. E.g., the prevalent strain in the RF in the epidemic season 2011-2012 was rotavirus P[8]G4 (pic. 2) - it was revealed in 56\% of cases (the studies were conducted in 11 subjects of the Russian Federation); rotavirus gastroenteritis was caused by strain P[8]G1 rarer (30\%) [4].

Pic. 1. Spread of rotavirus strains in the world

Spread of rotavirus strains in the world: rotavirus strains in different geographic zones $(1996-2007)^{1}$

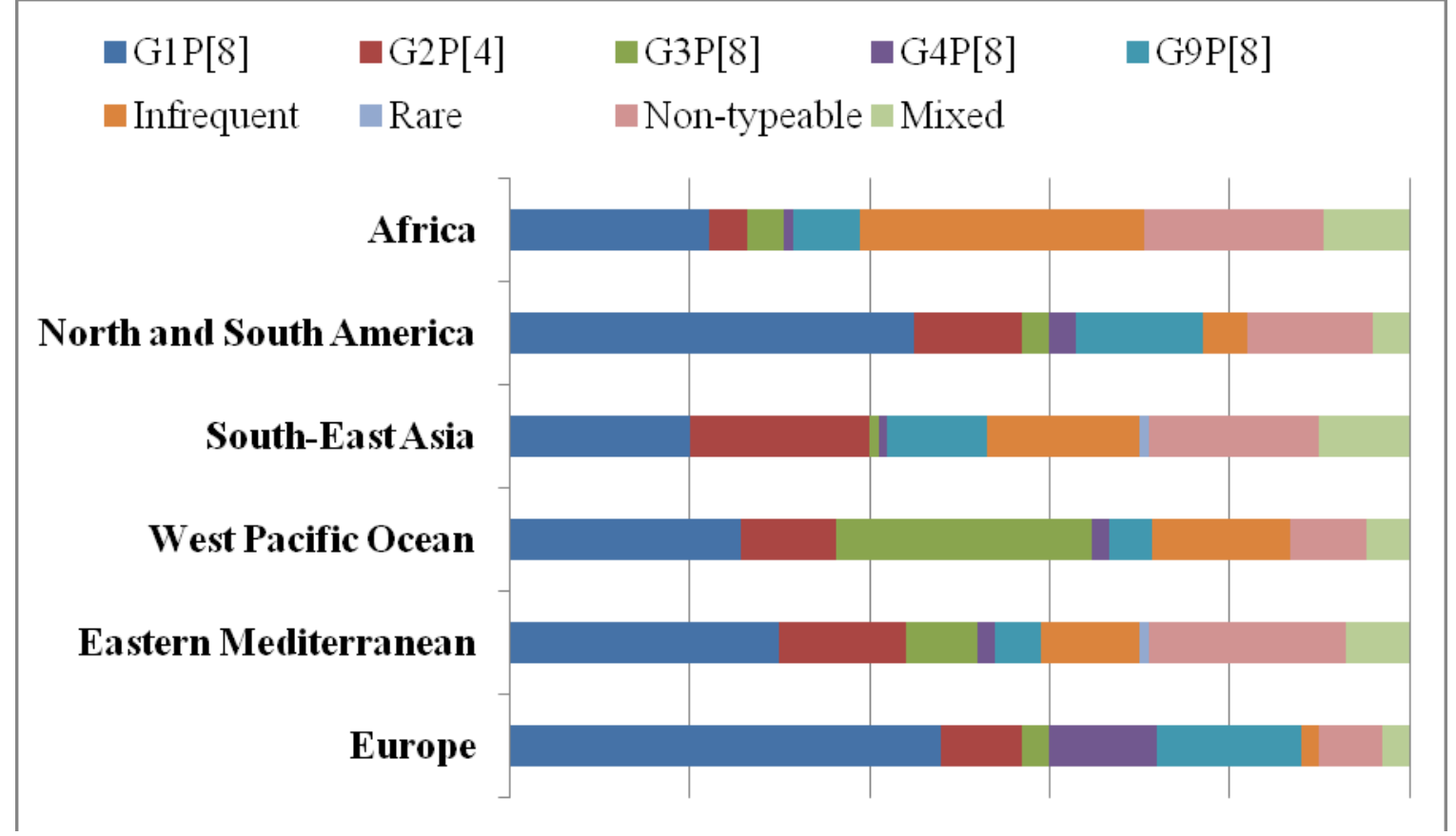

1. K. Banyal et al. Vaccine. 2012; 305: A122-A130.

Pic. 2. Dominant serotypes in the Russian Federation in 2011-2012.

Serotype G4 was dominant in the epidemic season 2011-2012 in the RF

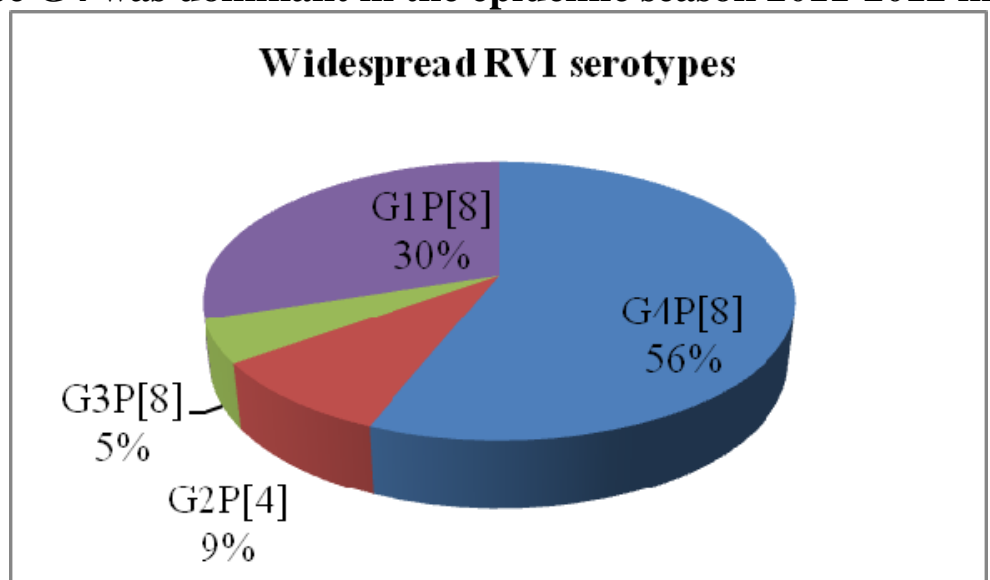

Among the hospitalized children under 5 years of age.

Epidemiology 
A source of the infection is an ill person or virus carrier. Transmission route - fecal-oral; however, the causative agent may be spread by air.

Rotavirus infection is especially dangerous in children of 6-24 months of age [5]. In children under 6 months of age this infection is usually asymptomatic due to the presence of protective maternal antibodies; due to the infection at an early age, serum antibodies to the virus appear in almost all children by 2-3 years of age (pic. 3) [6].

Pic. 3. Rate of rotavirus infection depending on the child's age

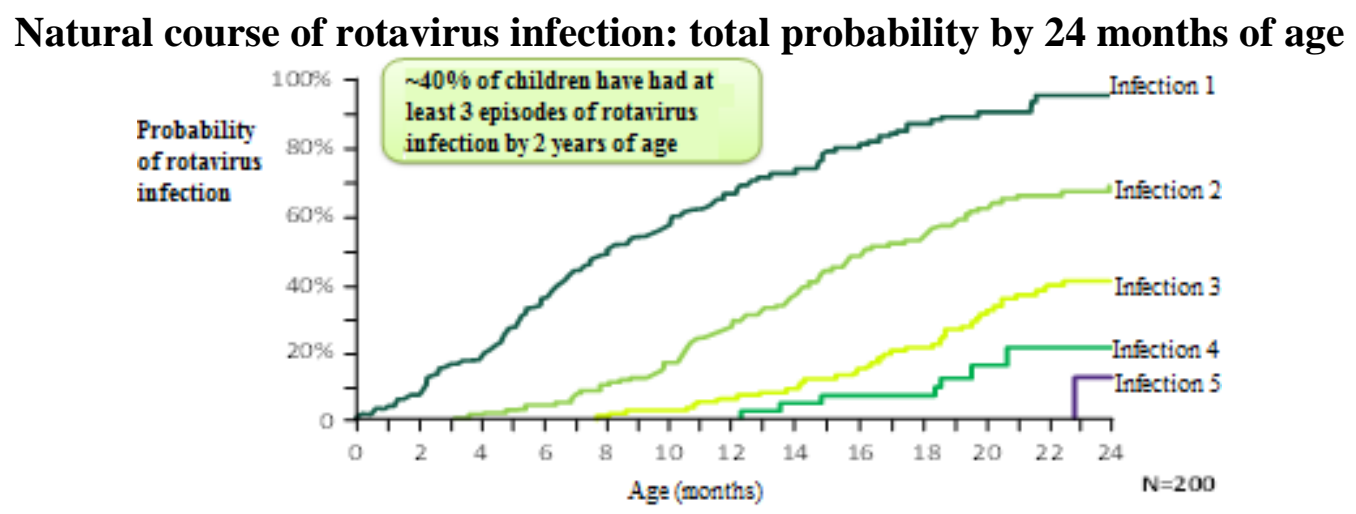

Rotavirus infection causes $126 \mathrm{mn}$. disease cases requiring outpatient observation and $2 \mathrm{mn}$. cases requiring hospitalization every year [7]. According to the calculation data, ca. $39 \%$ of hospitalizations due to liquid stool are connected with rotavirus infection. 500,000 death cases of the children under 5 years of age are also connected with rotavirus infection. Statistics about the number of RVGE episodes both in the poorest African countries and the developed European countries is the same [8].

The share of rotavirus gastroenterites in the Russian Federation is $44-47 \%$ of cases (according to the data for the latest 5 years). Up to $5 \%$ of infant deaths, or 86 cases per 100,000 fatal outcomes, in children under 5 years of age are connected with RVGE $[9,10]$.

\section{Clinical presentation of the disease}

The disease's incubation period varies from 15 hours to 7 days (1-2 days on the average). The disease has an acute onset. Body temperature reaches $\geq 37.9^{\circ} \mathrm{C}$ in most hospitalized children; in some children it may increase up to and exceed $39^{\circ} \mathrm{C}$. If the disease is mild, intense fever is observed neither in adults not in children. Patients mention pains in the epigastric area, nausea and emesis. Examination often reveals pharyngeal hyperemia, symptoms of rhinitis and expansion of cervical lymph nodes. Symptoms of lesion of digestive organs, primarily of the small intestine, are considered the most typical manifestations of the disease.

The disease is distinguished by liquid watery stool (up to 10-20 times per day) with sharp smell; sometimes stool is slightly turbid and whitish; it may resemble stool of a patient with cholera [4]. It is also distinguished by loud borborygmus. Urges to defecation are imperative; no false urges. Admixture of mucus and blood in stool is observed in some patients; this indicates combination of the rotavirus disease with a bacterial infection (shigellosis, escherichiosis, campilobacteriosis). Fever and general intoxication are more intense in these patients. Upper respiratory tract's inflammation symptoms are revealed in some patients with rotavirus diseases; some authors consider them to be caused by the secondary viral infection.

Dehydration may develop in case of abundant liquid stool and abundant emesis. Dehydration is observed rather often (in $75-85 \%$ of the hospitalized children). Loss of $8 \%$ of body weight with liquid, which is probable at this infection, is life-threatening. In case emesis is infrequent and liquid intake through the mouth is possible, the child may be observed outpatiently. However, children often refuse taking liquid and/or the drunk liquid cannot be kept in the body due to high 
vomiting reflex. In such cases, liquid may only be compensated by means of intravenous dropby-drop introduction - the child has to be hospitalized to the contagious isolation ward.

The later stages are characterized by the development of severe dehydration with decompensated metabolic acidosis develops; renal failure and hemodynamic disorders may occur.

\section{Methods of prevention of rotavirus infection}

Rotavirus infection is a highly contagious disease, which may be transmitted by water, food and regular contact. Rotavirus infection's causative agents are distinguished by high resistance. Rotavirus may survive outside of a human body for a long time - in water, food and the objects infected by patients' discharge.

Measures of non-specific prevention of RV-infection are based on the observance of hygiene and sanitary norms: washing of hands, use only of boiled water for drinking, tap water purification and chlorination. In case an episode of rotavirus infection occurs in a family, the ill person must be isolated and provided with personal tableware and linen.

However, practice shows that the aforementioned measures do not lead to any considerable reduction in the number of cases of RV-infection; that is why vaccination is the most reliable method of its specific prevention.

In order to develop the vaccines, rotavirus capability of recombination of genetic material was employed. The first experience of using the vaccine based on the rotavirus of rhesus monkeys, was not successful: cohort immunization of children with vaccine Rotashield (Wyeth) started in the USA in 1998; however, the vaccination was terminated after 1 year of application due to the infrequent ( 1 case per 10,000 vaccinated children), but life-threatening side effect - intestinal invagination [10].

2 types of vaccines for prevention of rotavirus infection are present in the pharmaceutical market at the moment: pentavalent vaccine Rotateq (MSD) and monovalent vaccine Rotarix (GSK). Both vaccines are live, prescribed for ingestion and applied to infants. However, vaccines differ in the manufacturing principle and type composition. Vaccine Rotateq is registered in the Russian Federation at the moment.

\section{Vaccine Rotarix}

Rotarix is a live attenuated rotavirus vaccine based on strain 89-12 (G1P[8]). The vaccine is used to prevent RVGE caused by viruses G1, G3, G4 and G9; its administration starts at the age of 6; the second administration occurs 4-6 weeks after the first one and before 24 weeks of age.

Results of the conducted 2-year-long observations showed that efficacy of this vaccine against severe forms of gastroenterites is $90.4 \%$, against rotavirus gastroenteritis of any severity $-78.9 \%$ [10].

This vaccine is not registered in the Russian Federation.

\section{Vaccine Rotateq}

Rotateq is a pentavalent vaccine; it contains 5 reassortant human and bovine viruses $(\mathrm{G} 1, \mathrm{G} 2$, G3, G4 and P1A[8]). It also contains adjuvants: sucrose, sodium citrate, dihydrate, monosubstituted sodium phosphate, monohydrate, sodium hydroxide, polysorbate 80 , rotavirus solvent and nutrient medium LKPM-3. Outward appearance: transparent yellowish liquid; it may sometimes have a shade of pink. It is issued in $2 \mathrm{ml}$ tubes for oral administration containing 1 inoculative dose [11]. It is used to prevent rotavirus gastroenteritis caused by rotaviruses of serotypes G1, G2, G3, G4 and the serotypes G containing P1A[8] in children of 6-32 weeks of age.

Contraindications to the prescription of rotavirus vaccine are: either high sensitivity to the vaccine's components or reaction to the previous dose, malformations of the gastrointestinal 
tract, invagination in anamnesis, either immunodeficiency or suspicion of immunodeficiency and HIV-carriage, acute form of diarrhea or emesis, acute inflammatory processes accompanied by high body temperature and either fructose intolerance or insufficiency of sucrose and isolmaltose enzymes [11].

Vaccination scheme: the $1^{\text {st }}$ dose of the vaccine is to be administered at the age of 6-12 weeks, the $2^{\text {nd }}$ dose $-4-6$ weeks after the first one, the $3^{\text {rd }}$ dose - before 32 weeks of age. As the vaccine is compatible with all the vaccines included in the National vaccination calendar of the RF (DTP, IPV or OPV, Hib), there are various vaccination schemes:

- 1.5 months of age -3 months -4.5 months;

- 2 months of age -3 months -4.5 . months;

- 3 months of age -4.5 . months -6 months;

- 4.5 months of age -6 months -7 months;

- other variants.

Vaccination against rotavirus infection is also indicated for premature infants born after the $25^{\text {th }}$ gestation week. Efficacy and safety of the vaccination in premature infants was also proved by clinical trials [12].

The vaccine is highly immunogenic and causes $90-95 \%$ increase in antibodies in blood serum after the 3-step immunization scheme in comparison with the initial level.

According to the trials, efficacy of vaccination against severe cases of RVGE is $98 \%$, against any forms of RV-gastroenterites - 74\%. According to the trials conducted by the REST (placebocontrolled trial of efficacy and safety), hospitalization risk decreased by $96 \%$, number of visits to emergency units - by $94 \%$, to doctors - by $86 \%$ after the completed immunization scheme.

Clinical trials confirmed that a complete 3 -step vaccination course is required to achieve necessary protection and duration of immunity against rotavirus infection. However, the trials showed that the number of severe cases of RVGE requiring hospitalization decreased considerably after administration of the $1^{\text {st }}$ dose of the rotavirus vaccine.

This vaccine has a high safety profile. Occurrence of severe undesirable reactions was being assessed in 36,150 vaccinated children and 35,536 children of the placebo group within 42 days after intake of each consecutive dose. The rate of undesirable reactions was $0.1 \%$ in the group of vaccinated children and $0.2 \%$ in the placebo group [11].

3 joint clinical trials were taken into consideration to assess undesirable phenomena $(6,130$ vaccinated children and 5,560 children of the placebo group).

The most frequent undesirable phenomena (1/10) registered after application of the pentavalent rotavirus vaccine (more often observed in the group of vaccinated children $-0.02-2.6 \%$ ) were upper respiratory tract infections, diarrhea, emesis, acute otitis media, irritability and cough (tb. 1).

Kawasaki disease was observed in 5 out of 36,150 children $(0.014 \%$ of cases $)$ in the group of vaccinated children and in 1 out of 35,536 children $(0.002, p>0.005)$ in the placebo group. These data do not confirm the increased risk of Kawasaki disease resulting from the immunization with vaccine Rotateq.

42-day-long observation of patients after each consecutive dose revealed intestinal invagination in 6 out of 34,837 patients, who had received vaccine Rotateq, and in 5 out of 34,788 patients of the placebo group [11]. Thus, no connection of vaccination with the pentavalent vaccine and intestinal invagination development was observed.

\section{Conclusion}

Vaccines Rotateq and Rotarix are registered abroad and are successfully used in Australia, Belgium, the USA, Finland, Germany and other countries. The data on decrease in the number of RVGE-associated hospitalizations are as follows: in Belgium - by $83-90 \%$, in the USA - by $86 \%$, in Finland - by $80 \%$, in Australia - by $93 \%$ [13]. 
The vaccination in foreign countries conducted in the framework of the national calendars allowed reducing burden of rotavirus infection in Central and Eastern Europe.

Reduction in the number of hospitalizations due to rotavirus gastroenteritis, significant reduction in hospitalization rate in the cold season, the number of visits to doctors and the number of workdays missed by parents due to their children's diarrhea are possible after the pentavalent rotavirus vaccine is registered in the Russian Federation.

The World Health Organization recommends introducing vaccination against rotavirus infection in the National vaccination calendar and considering this high-priority prevention [14, 15]. It should be noted that vaccination of the risk groups only does not have any considerable use to the public health in general. Maximal coverage must be achieved when introducing vaccination against rotavirus infection. Only introduction of the rotavirus vaccine into the national immunization programs can attain reduction in RVGE morbidity and the number of hospitalizations and infant deaths of this infection.

Lack of data on circulating serotypes must not be a hindrance to the introduction of an RVvaccination program [14].

Table 1. Undesirable reactions to administration of the vaccines observed within 42 days after each consecutive dose.

\begin{tabular}{|c|c|c|}
\hline $\begin{array}{c}\text { Undesirable } \\
\text { reactions }\end{array}$ & $\begin{array}{c}\text { Pentavalent rotavirus vaccine } \\
\mathbf{( n = 6 , 1 3 8 ) ,} \mathbf{\%}\end{array}$ & $\begin{array}{c}\text { Placebo group (n=5,537), } \\
\mathbf{\%}\end{array}$ \\
\hline Diarrhea & 24.1 & 21.3 \\
\hline Emesis & 15.2 & 13.6 \\
\hline Otitis media & 14.5 & 13.0 \\
\hline Cough & 10.5 & 10.1 \\
\hline Nasopharyngitis & 6.9 & 5.8 \\
\hline
\end{tabular}

\section{REFERENCES}

1. Arvin A. M., Greenberg H. B. Virology. 2006; 344: 240-249.

2. Centers for disease control and Prevention. Morb Mortal Wkly Rep. 2006; 55 (RR-12): 1-13.

3. Banyia K. et al. Vaccine. 2012; 30S: A122-A130.

4. Podkolzin A. T. et al. Hospital-Based Surveillance of Rotavirus and other Viral Agents of Diarrhea in Children and Adults in Russia, 2005-2007. The Journal of Infectious Diseases. 2009; 200: S228-33.

5. Centers for disease control and Prevention. Morb Mortal Wkly Rep. 2009; 58 (RR-2): 1-25.

6. Velazquez F. R. et al. N Engl J Med. 1996; 335: 1022-1028.

7. Isla Ogilvie, Hanane Khoury, Antoine C., El Khoury et al. Burden of rotavirus gastroenteritis in the pediatric population in Central and Eastern Europe. Human Vaccines. 2011 May; 7 (5): 523-533.

8. Weekly epidemiological record. 2008; 47: 421-425.

9. Available at: http://www.rospotrebnadzor.ru

10. Immunoprofilaktika-2011. 3-e izd. Pod red. V. K. Tatochenko, N. A. Ozeretskogo, A. M. Fedorova [Immunization-2011. $3^{\text {rd }}$ ed. Edited by V.K. Tatochenko, N.A. Ozeretskii, A.M. Fedorov]. Moscow, IPK "Content-press", 2011.

11. Available at: http://www.rotateq.com

12. Goveia M. G. et al. Safety and efficacy of the pentavalent human-bovine (WC3) reassortant rotavirus vaccine in healthy premature infants. Pediatr Infect Dis J. 2007; 26: 1099-1104.

13. The results of the 31-st Annual Meeting of the European Society for Paediatric Infectious Diseases (ESPID). Milan, Italy. May 28 June 1. 2013. 
14. Rotavirus vaccines. WHO position paper. 2013 Jan 5; 88: 49-64. Available at: http://www.who.int/wer

15. Namazova-Baranova L.S. Pediatricheskaya farmakologiya - Pediatric pharmacology. 2012; 9 (4): $15-24$. 